\title{
Transient growth in thermocapillary liquid layers
}

\author{
Kai-Xin $\mathrm{Hu},{ }^{1,}{ }^{*}$ Sheng Zheng, ${ }^{1}$ and Qi-Sheng Chen ${ }^{2,3}$ \\ ${ }^{1}$ Key Laboratory of Impact and Safety Engineering, Ministry of Education, School of Mechanical Engineering \\ and Mechanics, Ningbo University, Ningbo, Zhejiang 315211, China \\ ${ }^{2}$ School of Engineering Science, University of Chinese Academy of Sciences, Beijing 100190, China \\ ${ }^{3}$ Key Laboratory of Microgravity, Institute of Mechanics, Chinese Academy of Sciences, Beijing 100190, China
}

(Received 4 July 2019; published 6 January 2020)

\begin{abstract}
The transient growth in thermocapillary liquid layers is examined by nonmodal stability theory. Two kinds of thermocapillary liquid layers including linear flow and return flow are considered. The transient growth is measured by a growth function, which depends on both the velocity and temperature. It was found that rather large transient growth occurs in subcritical flows at small Prandtl numbers (Pr), while the temperature field on the surface has a negative effect on the transient growth. In particular, the transient growth function increases significantly with the Reynolds number, but decreases with Pr. The most amplified perturbation is characterized by counter-rotating vortices and streaks, which are both nearly streamwise. Energy analysis shows that the energy of transient growth mainly comes from the basic flow, while the work done by Marangoni forces on the surface is negligible.
\end{abstract}

DOI: 10.1103/PhysRevFluids.5.014001

\section{INTRODUCTION}

Thermocapillary convection refers to the flow driven by the temperature-induced surface tension gradient. It appears in many practical applications, such as crystal growth [1], fusion welding [2], ink-jet printing [3], droplet migration [4], and nanofluid flows [5]. The thermocapillary flow has been studied over the years, and the related works have been reviewed by Davis [6] and Schatz and Neitzel [7]. Recently, the studies of thermocapillary convections have been extended to the flows in different geometries, such as a sphere [8], vertical cylinders [9], and horizontal cylinders [10]. The thermocapillary flows with two gas-liquid interfaces have been investigated by both experiments $[11,12]$ and numerical simulations [13,14].

The instability of thermocapillary convection has been studied extensively. Smith and Davis [15] have performed linear stability analysis on thermocapillary liquid layers. They claimed that there are two kinds of instabilities, which are stationary longitudinal rolls and unsteady hydrothermal waves. Chan and Chen [16] have examined the stability of thermocapillary fluid layers coupled with gravity effect. Their results agree with the experiment by Riley and Neitzel [17]. In Refs. [15,16], the surface tension was considered to be large enough, so the model of liquid layer with a nondeformable surface was used. In more general cases, some authors have found that the surface deformation is also important to thermocapillary instabilities [18-20].

Recently, three-dimensional numerical simulations have been performed by Zhang et al. [21] on the thermocapillary convection in a shallow annular pool, and the effect of surface heat dissipation has been demonstrated. Davalos-Orozco [22] has studied the sideband thermocapillary instability of a thin liquid film flowing down the outside of a hot vertical cylinder. Kang et al. [23] have reported the space experimental results on the thermocapillary convection in an open annular liquid

\footnotetext{
*Corresponding author: hukaixin@nbu.edu.cn
} 
pool. It is worth noting that the studies of thermocapillary instabilities have been generalized to liquid layers on an inclined plane [24] and non-Newtonian fluids, such as viscoelastic fluids [25,26], shear-thinning fluids [27], and viscoplastic fluids [28].

In the theoretical studies of thermocapillary instabilities, the modal analysis is mostly used, where a small perturbation is assumed to vary exponentially with time. This method can be used to predict the long time behavior of disturbances. However, in many cases, the short-term characteristics of flow instabilities are very important in the transition to turbulence. For this reason, some authors have turned to the nonmodal stability theory $[29,30]$.

Trefethen et al. [31] have investigated the transient energy growth and the transient behavior of response to external excitations for plane Poiseuille and Couette flows. The concept of nonmodal stability theory addressed by them has been used to study the energy growth for channel flows [32] and pipe flows [33]. These works suggested that there can be substantial transient growth even if the flow is linearly stable in the normal-mode analysis. Nouar et al. [34] have examined modal and nonmodal linear stability of the plane Bingham-Poiseuille flow, which shows that the optimal disturbance depends on the Bingham number. The nonmodal instability in plane Couette flow of a power-law fluid has been examined by Liu and Liu [35]. The result shows that the shear-thinning effect significantly increases the amplitude of response to external excitations and initial conditions. Balestra et al. [36] have performed an optimal transient growth analysis for the Rayleigh-Taylor instability under curved substrates. They found that the system displays a linear transient growth potential that gives rise to two different scenarios depending on the value of the Bond number.

The nonmodal stability of thermocapillary convection has not been thoroughly discussed in previous works. However, in thermocapillary liquid layers, the critical Reynolds numbers are on the order of 1000 at small Pr. The energy growth can reach $O(1000)$ in channel flows [32,35] at these Reynolds numbers, so one would expect that there may also be large transient growth in thermocapillary convections. In the present work, the transient growth of thermocapillary convection is investigated by nonmodal linear stability analysis. The model of thermocapillary liquid layers is considered. The flows at different Prandtl numbers and Biot numbers are discussed.

The paper is organized as follows. In Sec. II, the physical model and mathematical formulations are presented. The governing equations for the flow and perturbations are derived. Then in Sec. III, the transient growth function is determined. The level lines of maximum growth and the optimal perturbation flow fields are displayed. After that, the instability mechanism is discussed in Sec. IV. Finally, we summarize the results and present the conclusions in Sec. V.

\section{PROBLEM FORMULATION}

We consider the model of thermocapillary liquid layer proposed by Smith and Davis [15], where a fluid layer above an infinite rigid plane is set in motion by a temperature gradient imposed on its free surface (see Fig. 1). The surface tension $\sigma^{\prime}$ is linearly related to the temperature $T$ : $\sigma^{\prime}=\sigma_{0}^{\prime}-\gamma\left(T-T_{0}\right)$, where $\gamma$ is the variation of surface tension with temperature. In most cases, the surface tension decreases with the temperature; thus $\gamma=-d \sigma^{\prime} / d T>0$. The convection is driven by the thermocapillary force. $x, y$, and $z$ are the streamwise, spanwise, and wall-normal direction, respectively. This model has been widely used in the theoretical study of thermocapillary instabilities. The oblique hydrothermal waves predicted by this model have been observed in experiment [17] and numerical simulations [37]. There are two kinds of flows, which are linear flow and return flow. They will be discussed later.

\section{A. Governing equations}

The dimensionless parameters are defined as follows. $R$ is the Reynolds number $R=\rho \hat{U}_{0} d / \mu$, where $\mu$ is the viscosity, $\rho$ is the fluid density, and $\hat{U}_{0}$ is the characteristic velocity with the expression $\hat{U}_{0}=b \gamma d / \mu$. Here, $b$ is the temperature gradient on the surface, Ma is the Marangoni number defined as $\mathrm{Ma}=b \gamma d^{2} /(\mu \chi)$, and $\chi$ is the thermal diffusivity. Ma and $R$ have the relation $\mathrm{Ma}=R \operatorname{Pr}$, where $\operatorname{Pr}$ is the Prandtl number; $\operatorname{Pr}=\mu /(\rho \chi)$. 

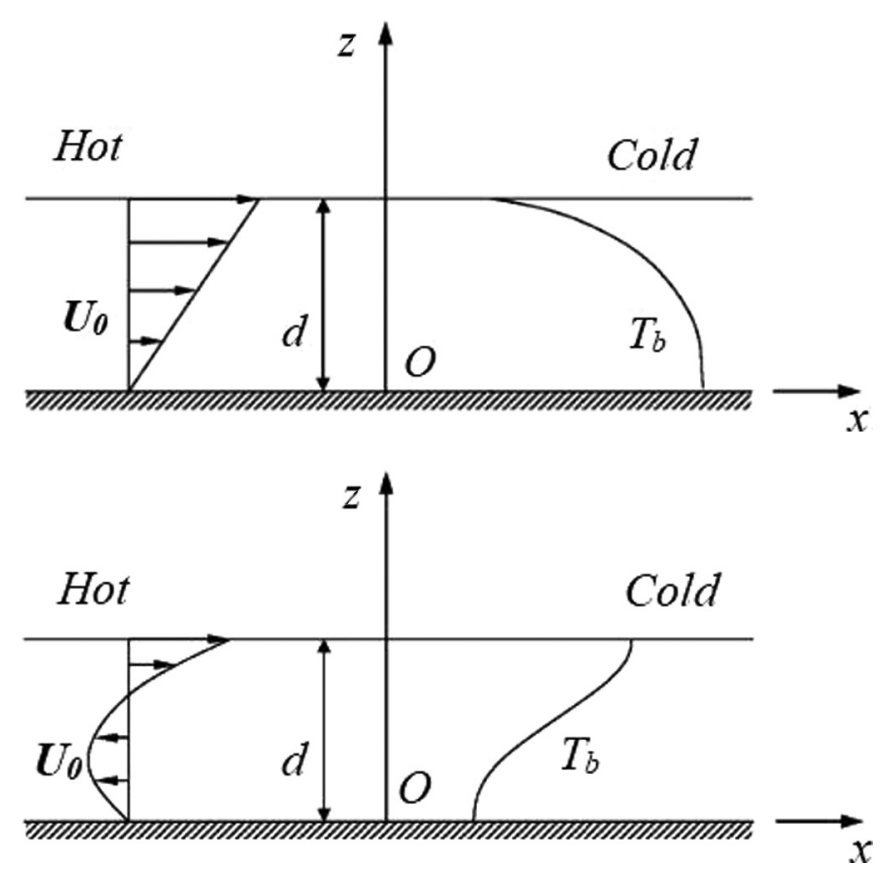

FIG. 1. Schematic of thermocapillary liquid layers: (a) linear flow; (b) return flow. Here, $d$ is the depth of the layer, $T_{b}$ is the temperature distribution in the vertical direction, and $U_{0}$ is the velocity field.

The magnitude of the surface deformation can be measured by the capillary number $[15,17]$ : $\mathrm{Ca}=\mu \hat{U}_{0} / \hat{\sigma}$, where $\hat{\sigma}$ is the characteristic surface tension. We assume that the free surface is nondeformable in the following. For liquid silicon [15] and silicone oil [17], $O(\mathrm{Ca}) \approx 10^{-3} \ll 1$, so the assumption of a nondeformable free surface can be satisfied.

Below we give the dimensionless governing equations, which are the continuity equation, the momentum equation, and the energy equation, respectively.

$$
\begin{aligned}
\nabla \cdot \mathbf{u} & =0 \\
R\left(\frac{\partial \mathbf{u}}{\partial t}+\mathbf{u} \cdot \nabla \mathbf{u}\right) & =-\nabla p+\nabla \cdot \boldsymbol{\tau} \\
\frac{\partial T}{\partial t}+\mathbf{u} \cdot \nabla T & =\frac{1}{\mathrm{Ma}} \nabla^{2} T .
\end{aligned}
$$

Here, $\mathbf{u}, p$, and $T$ stand for the velocity, pressure, and temperature, respectively. $\boldsymbol{\tau}$ is the stress tensor. We assume the fluid is Newtonian, so its constitutive equation is

$$
\boldsymbol{\tau}=\mathbf{S}
$$

where $\mathbf{S}=\nabla \mathbf{u}+(\nabla \mathbf{u})^{\mathrm{T}}$ is the strain-rate tensor. The viscous dissipation is ignored in (2.3). The boundary conditions are set as follows:

$$
\begin{gathered}
\mathbf{u}=(u, v, w)=0, \quad \frac{\partial T}{\partial z}=0, \quad z=0, \\
\tau_{13}+\frac{\partial T}{\partial x}=0, \quad \tau_{23}+\frac{\partial T}{\partial y}=0, \quad w=0, \quad-\frac{\partial T}{\partial z}=\operatorname{Bi}\left(T-T_{\infty}\right)+\tilde{Q}, \quad z=1 .
\end{gathered}
$$


In (2.5), there is no slip and zero heat flux on the rigisd plane. In (2.6), the stress on the surface is caused by the thermocapillary effect. $T_{\infty}$ is the temperature of the bounding gas far from the surface. $\mathrm{Bi}=\hat{h} d / \hat{k}$ is the Biot number, where $\hat{h}$ is the unit thermal surface conductance, and $\hat{k}$ is the thermal conductivity. $\tilde{Q}$ is the imposed heat flux to the environment, which can be determined by the form of basic flow [15].

The basic flow is fully developed while its temperature is linear in $x$ as imposed due to $\tilde{Q}$ plus a distribution in $z$ as follows:

$$
\mathbf{u}=\left[U_{0}(z), 0,0\right], \quad T_{0}(x, z)=-x+T_{b}(z) .
$$

Two kinds of flows are investigated. The first kind is the linear flow, whose velocity is linear in $z$,

$$
U_{0}(z)=z, \quad T_{b}(z)=\operatorname{Ma} \frac{1}{6}\left(1-z^{3}\right) .
$$

The second kind is the return flow, which has zero mass flux in the vertical section $\int_{0}^{1} U_{0} d z=0$, so

$$
U_{0}(z)=\frac{3}{4} z^{2}-\frac{1}{2} z, \quad T_{b}(z)=\operatorname{Ma}\left(-\frac{1}{16} z^{4}+\frac{1}{12} z^{3}-\frac{1}{48}\right)
$$

The streamwise velocity $U_{0}$ only depends on $z$; thus the continuity equation (2.1) is automatically satisfied. The basic flow is steady and parallel, so the momentum equation (2.2) can be satisfied, and the pressure gradient has $\frac{d p}{d x}=\frac{d^{2} U_{0}}{d z^{2}}$, which is constant for both the return and linear flows. Applying (2.7) to the energy equation (2.3), we have $-U_{0}=\frac{1}{\mathrm{Ma}} \frac{d^{2} T_{b}}{d z^{2}}$. It is obvious that the solutions in (2.8) and (2.9) satisfy this equation. The distributions of velocity and temperature are displayed in Fig. 1.

\section{B. Modal analysis}

First, we study the flow stability by modal analysis. Suppose an infinitesimal perturbation in the normal-mode form is added to the basic flow,

$$
\begin{aligned}
(\mathbf{u}, T, P, \boldsymbol{\tau}) & =\left(\mathbf{u}_{0}, T_{0}, P_{0}, \boldsymbol{\tau}_{0}\right)+\boldsymbol{\delta}, \\
\boldsymbol{\delta} & =(\widehat{u}, \widehat{v}, \widehat{w}, \widehat{T}, \widehat{P}, \widehat{\boldsymbol{\tau}}) \exp [i(-\omega t+\alpha x+\beta y)] .
\end{aligned}
$$

The subscript 0 stands for the basic flow and hereafter, the variables without subscript 0 stand for the perturbation. Here, $\omega$ is the complex frequency; $\alpha, \beta$ are the wave number in the $x$ and $y$ directions, respectively. The wave number and the propagation angle are defined as $k=\sqrt{\alpha^{2}+\beta^{2}}$ and $\phi=\tan ^{-1}(\beta / \alpha)$, respectively.

The boundary conditions of perturbations are determined as follows.

$$
\begin{aligned}
\widehat{u} & =\widehat{v}=\widehat{w}=D \widehat{T}=0, \quad z=0, \\
\widehat{\tau}_{13}+i \alpha \widehat{T} & =0, \quad \widehat{\tau}_{23}+i \beta \widehat{T}=0, \quad \widehat{w}=0, \quad D \widehat{T}+\mathrm{Bi} \cdot \widehat{T}=0, \quad z=1 .
\end{aligned}
$$

Here, $D=\frac{d}{d z}$. Substituting (2.10) into governing equations (2.3)-(2.6), the linearized perturbation equations can be derived as follows:

$$
\begin{aligned}
& i \alpha \widehat{u}+i \beta \widehat{v}+D \widehat{w}=0, \\
& R\left[\beta\left(\widehat{w} D U_{0}+U_{0} i \alpha \widehat{u}\right)-\alpha\left(U_{0} i \alpha \widehat{v}\right)\right]-\beta\left(i \alpha \widehat{\tau}_{11}+i \beta \widehat{\tau}_{12}+D \widehat{\tau}_{13}\right)+\alpha\left(i \alpha \widehat{\tau}_{12}+i \beta \widehat{\tau}_{22}+D \widehat{\tau}_{23}\right) \\
& =\operatorname{Ri\omega }(\beta \widehat{u}-\alpha \widehat{v}), \\
& R \alpha\left(D \widehat{w} \cdot D U_{0}+\widehat{w} D^{2} U_{0}+D U_{0} \cdot i \alpha \widehat{u}+U_{0} i \alpha D \widehat{u}\right)+R \beta\left(D U_{0} \cdot i \alpha \widehat{v}+U_{0} i \alpha D \widehat{v}\right)-R i k^{2}\left(U_{0} i \alpha \widehat{w}\right) \\
& -\left(i \alpha^{2} D \widehat{\tau}_{11}+2 i \alpha \beta D \widehat{\tau}_{12}+\alpha D^{2} \widehat{\tau}_{13}+i \beta^{2} D \widehat{\tau}_{22}+\beta D^{2} \widehat{\tau}_{23}\right)+i k^{2}\left(i \alpha \widehat{\tau}_{13}+i \beta \widehat{\tau}_{23}+D \widehat{\tau}_{33}\right) \\
& =\operatorname{Ri\omega }\left(\alpha \widehat{D} \hat{u}+\beta D \widehat{v}-i k^{2} \widehat{w}\right) \text {, }
\end{aligned}
$$


TABLE I. The critical parameters of thermocapillary liquid layers.

\begin{tabular}{lcccccc}
\hline \hline Flow & Pr & Bi & Ma & $k$ & $\psi$ & $c$ \\
\hline Linear flow & 0.001 & 1 & 17 & 0.82 & $90^{\circ}$ & 0.0150 \\
Return flow & 0.01 & 0 & 6.3 & 0.24 & $77^{\circ}$ & 0.0736 \\
\hline \hline
\end{tabular}

$$
\begin{gathered}
\operatorname{Ma}\left(\widehat{u} \frac{\partial T_{0}}{\partial x}+\widehat{w} \frac{\partial T_{0}}{\partial z}+U_{0} i \alpha \widehat{T}\right)+\left(\alpha^{2}+\beta^{2}\right) \widehat{T}-D^{2} \widehat{T}=\operatorname{Ma} i \widehat{T}, \\
\widehat{\tau}_{11}-\mu 2 i \alpha \widehat{u}=0, \quad \widehat{\tau}_{12}-\mu(i \alpha \widehat{v}+i \beta \widehat{u})=0, \quad \widehat{\tau}_{13}-\mu_{t}(D \widehat{u}+i \alpha \widehat{w})=0, \\
\widehat{\tau}_{22}-\mu(2 i \beta \widehat{v})=0, \quad \widehat{\tau}_{23}-\mu(i \beta \widehat{w}+D \widehat{v})=0, \quad \widehat{\tau}_{33}-\mu(2 D \widehat{w})=0 .
\end{gathered}
$$

Then, the Chebyshev collocation method is used to solve the eigenvalue problem with the form of $\mathbf{W g}=\omega \mathbf{Z g}$, where $\mathbf{W}, \mathbf{Z}$ are two matrices, and $\mathbf{g}$ is the eigenvector [38]. The eigenvalues are obtained by using the QZ algorithm available in the MATLAB software package. We use more than 80 Chebyshev nodes to ensure the accuracy in the following.

In order to validate our code, we have computed the critical parameters of thermocapillary liquid layers. The results are listed in Table I, which agree with those in Ref. [15]. Here, $\boldsymbol{\Psi}=180^{\circ}-\phi$, and $c=\left|\sigma_{i}\right| / k$ is the wave speed.

\section{Nonmodal analysis}

Now we study the transient growth of perturbation by nonmodal stability analysis. Suppose a perturbation with the wave numbers $(\alpha, \beta)$ is the sum of normal modes as follows [32]:

$$
\boldsymbol{\Psi}(z, t)=\sum_{j} a_{j} \exp \left(-i \omega_{j} t\right) \boldsymbol{\Psi}_{j}(z)
$$

Here, $\boldsymbol{\Psi}(z, t)=(u, v, w, T)$ is a vector consisting of the perturbation velocity and temperature, the subscript $j$ stands for the $j$ th least stable mode, $a_{j}$ is the $j$ th expansion coefficient, and $\boldsymbol{\Psi}_{j}=$ $\left(\widehat{u}_{j}, \widehat{v}_{j}, \widehat{w}_{j}, \widehat{T}_{j}\right)$ is the $j$ th eigenvector. A disturbance "energy" can be defined as the following form [39],

$$
E=\|\boldsymbol{\Psi}\|^{2}=\int\left(|u|^{2}+|v|^{2}+|w|^{2}+\varsigma|T|^{2}\right) d z
$$

where $\varsigma$ is a positive coefficient. Then, the transient growth of perturbation can be measured by the growth function,

$$
G(t)=\sup _{\boldsymbol{\Psi}(0) \neq 0}\|\boldsymbol{\Psi}(t)\|^{2} /\|\boldsymbol{\Psi}(0)\|^{2}
$$

The transient growth function is obtained by using the algorithm available in the book of Schmid and Henningson [38]. In the computation, we only need to choose the first $K$ modes,

$$
\boldsymbol{\Psi}(z, t) \approx \sum_{j=1}^{K} a_{j} \exp \left(-i \omega_{j} t\right)\left(\widehat{u}_{j}, \widehat{v}_{j}, \widehat{w}_{j}, \widehat{T}_{j}\right) .
$$

The transient growth function is essentially independent of $K$ when $K$ is large enough [32],

$$
G(t) \approx\left\|\mathbf{F} \exp \left(-\mathrm{i} \boldsymbol{\Lambda}_{K} t\right) \mathbf{F}^{-1}\right\|_{2}^{2} .
$$


Here, $\boldsymbol{\Lambda}_{K}=\operatorname{diag}\left(\omega_{1}, \omega_{2} \cdots \omega_{K}\right) ; \mathbf{F}$ is a decomposition of a Hermitian matrix $\mathbf{A}=\mathbf{F}^{*} \mathbf{F}$, where $\mathbf{F}^{*}$ is the Hermitian conjugate of $\mathbf{F}$; and $\mathbf{A}$ is the matrix defined in terms of an inner product,

$$
A_{j l}=\left(\boldsymbol{\Psi}_{j}, \boldsymbol{\Psi}_{l}\right)=\int\left(\widehat{u}_{j}^{*} \widehat{u}_{l}+\widehat{v}_{j}^{*} \widehat{v}_{l}+\widehat{w}_{j}^{*} \widehat{w}_{l}+\varsigma \widehat{T}_{j}^{*} \widehat{T}_{l}\right) \cdot d z
$$

$\|\mathbf{H}\|_{2}$ is the 2-norm of the matrix $\mathbf{H}$, which is defined as $\|\mathbf{H}\|_{2}=\sup _{\kappa \neq 0}\|\mathbf{H} \kappa\|^{2} /\|\kappa\|^{2}$. Here $\kappa$ is a vector.

The choice of $\varsigma$ is arbitrary. However, we want the disturbance energy to be mainly a measure of kinetic energy as this seems more relevant to transition; thus

$$
\int\left(|u|^{2}+|v|^{2}+|w|^{2}\right) d z \gg \int \varsigma|T|^{2} d z
$$

However, when $\varsigma$ is too small, $G(t)$ will be singular at small $k$. We choose $\varsigma=k^{2} / 100$. The computation shows that the transient growth function is nearly independent of $\varsigma$ when $\varsigma$ is on the order of $O\left(k^{2} / 100\right)$. Therefore, the results below are not expected to change qualitatively.

\section{NUMERICAL RESULTS}

In the following, we compute the transient growth function $G(t)$ for subcritical flows, whose Marangoni number Ma is smaller than the critical value $\mathrm{Ma}_{\mathrm{c}}$ [15]. The maximum transient growth is defined as [32]

$$
G^{\max }=G\left(t^{\max }\right)=\max _{t \geqslant 0} G(t)
$$

Then, the optimal growth is defined as

$$
G^{\mathrm{opt}}=\max _{\alpha, \beta} G\left(t^{\mathrm{max}}\right),
$$

and $t^{\mathrm{opt}}$ is the time corresponding to $G^{\mathrm{opt}}$. The transient growth function, maximum growth, and optimal growth will be discussed in Secs. III A, III B, and III C, respectively. The computation shows that the transient growth function decreases obviously with $\operatorname{Pr}$. When $\operatorname{Pr}>0.1$, the largest transient growth is less than 10 for subcritical flows. Therefore, we restrict our attention to $\operatorname{Pr}=0.01$ and $\operatorname{Pr}=0.001$. Due to symmetry, we shall confine ourselves to the case $\alpha \geqslant 0, \beta \geqslant 0$.

\section{A. Transient growth function}

We plot the variation of transient growth function $G(t)$ with time for linear flow in Fig. 2. For $\operatorname{Pr}=0.001, \mathrm{Bi}=1$, the critical Marangoni number of linear flow is $\mathrm{Ma}_{\mathrm{c}} \approx 17$ [15]. The Reynolds numbers in Fig. 2 are far below the critical value $R_{c}=\mathrm{Ma}_{c} / \mathrm{Pr} \approx 17000$. However, large transient growth occurs in the subcritical flow.

It can be seen that the perturbation experiences a transient growth and then decays when the time is large enough. In Fig. 2(a), both $G(t)$ and $t^{\max }$ increase significantly with $R$. The largest transient growth is reached when the wave number is moderate [see Fig. 2(b)]. In Fig. 2(c), the maximum transient growth is relatively large for streamwise-independent $(\alpha=0)$ perturbation, and it decreases significantly with the decrease of $\phi$. This can also be seen in the level lines of the maximum growth in Sec. III B. $t^{\max }$ increases with $\phi$ obviously. In Fig. 2(d), $G(t)$ is not sensitive to Pr when $t<20$. However, both $G^{\max }$ and $t^{\max }$ decrease obviously with the increase of Pr. This suggests that the transient growth of thermocapillary convection depends on the temperature field, so it differs from those of channel flows. Furthermore, Fig. 2(e) shows that $G^{\max }$ increases with Bi.

The variations of transient growth function with time for return flow are displayed in Fig. 3. For $\operatorname{Pr}=0.001, \mathrm{Bi}=1$, the critical Marangoni number of return flow is $\mathrm{Ma}_{\mathrm{c}} \approx 7.2$, and the critical 

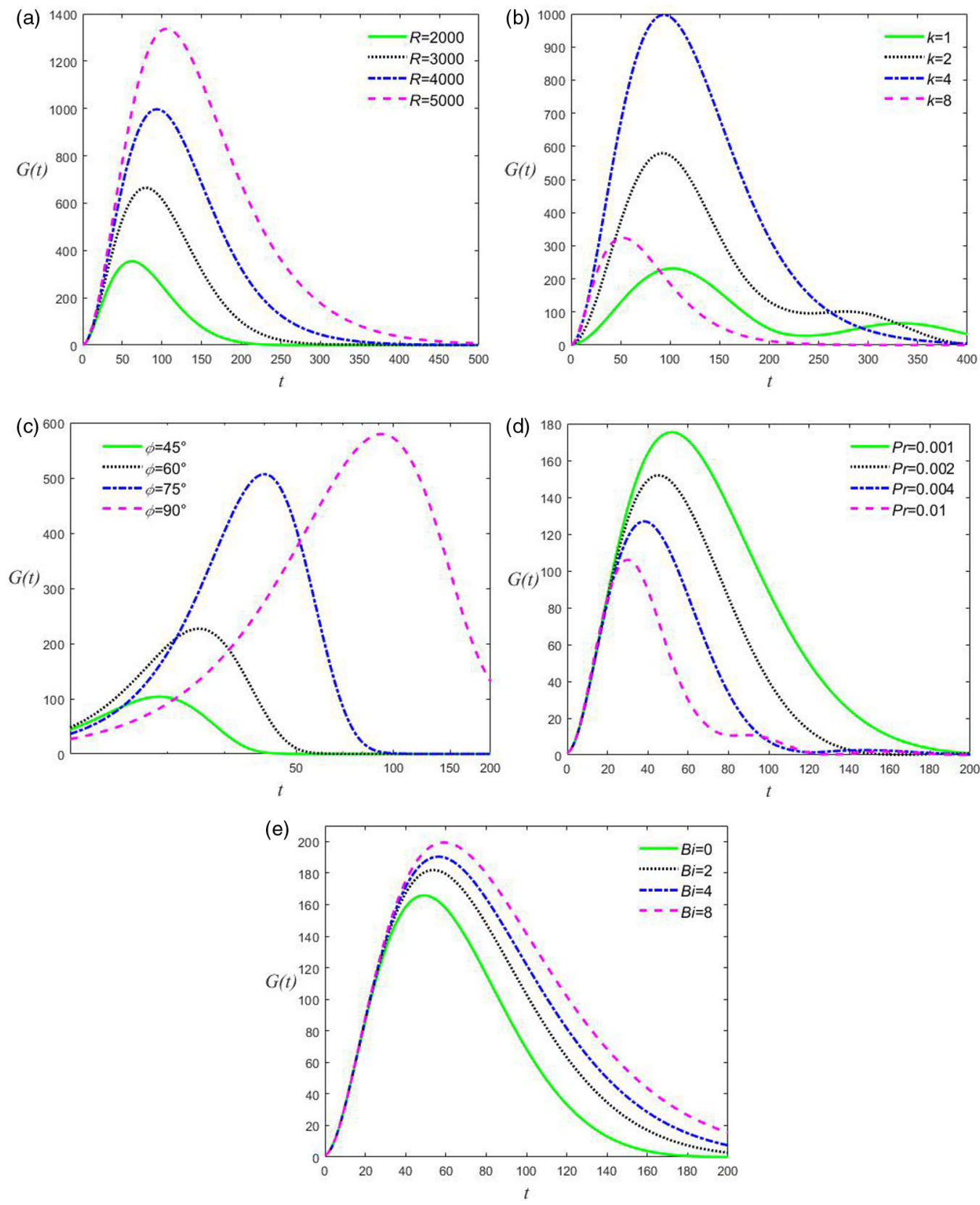

FIG. 2. The variation of $G(t)$ with time for linear flow: (a) at $\operatorname{Pr}=0.001, \mathrm{Bi}=1, k=4, \phi=90^{\circ}$ with various Reynolds numbers $R$; (b) at $\mathrm{Ma}=4, \mathrm{Pr}=0.001, \mathrm{Bi}=1, \phi=90^{\circ}$ with various wave numbers $k$; (c) at $\mathrm{Ma}=4, \operatorname{Pr}=0.001, \mathrm{Bi}=1, k=2$ with various propagation angles $\phi$; (d) at $R=1200, \mathrm{Bi}=1, k=3$, $\phi=90^{\circ}$ with various Prandtl numbers $\operatorname{Pr}$; (e) at $\mathrm{Ma}=1.2, \operatorname{Pr}=0.001, k=3, \phi=90^{\circ}$ with various Biot numbers Bi.

Reynolds number is $R_{c}=\mathrm{Ma}_{\mathrm{c}} / \mathrm{Pr} \approx 7200$; these are larger than those in Fig. 3. It can be seen that $G^{\max }$ increases with $R$ significantly, while $t^{\max }=O(100)$ in Fig. 3(a). Both $G^{\text {max }}$ and $t^{\max }$ decrease rapidly with the decrease of $\phi$ [Fig. 3(c)]. In plane channel flows, the maximum transient growth 

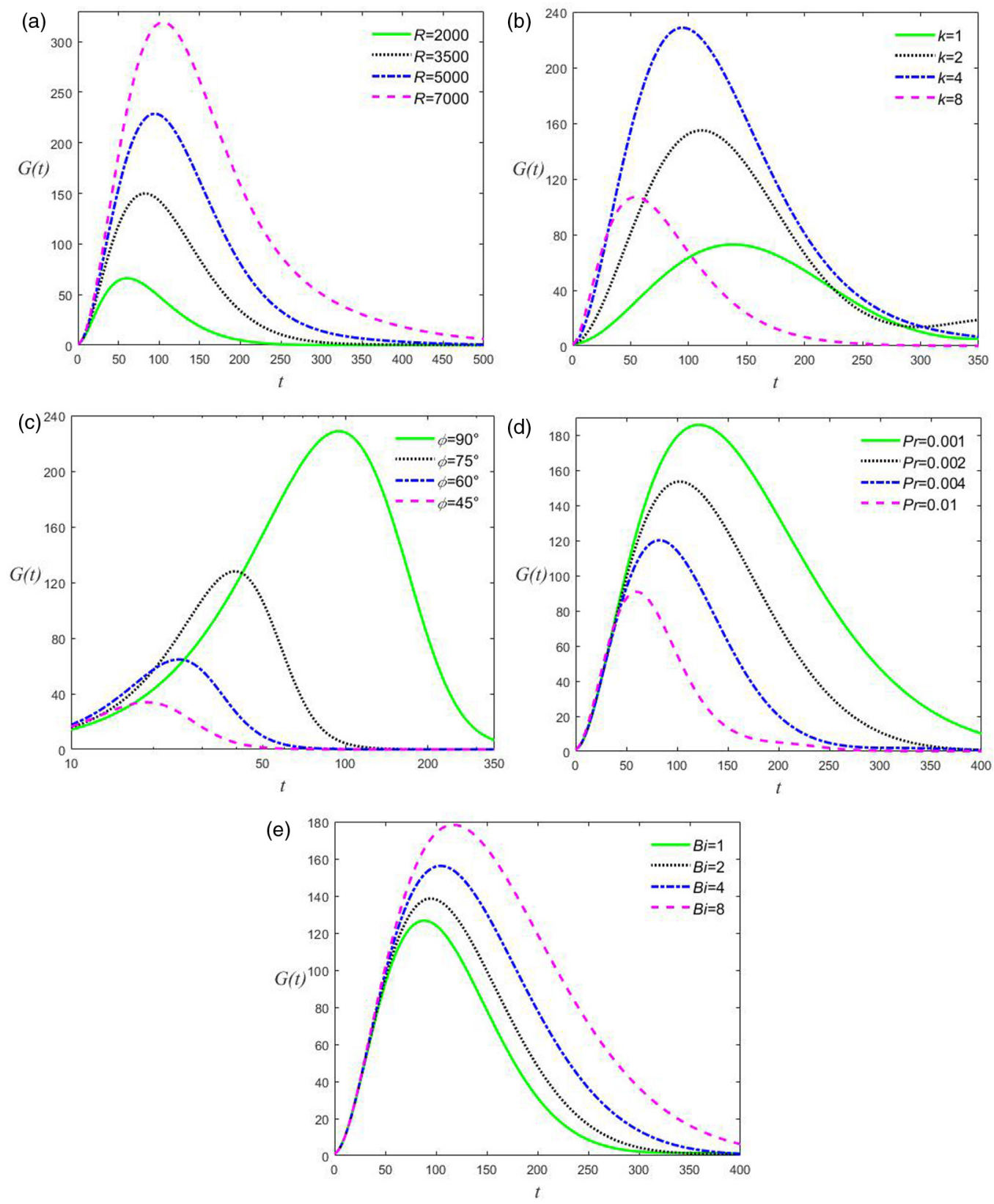

FIG. 3. The variation of $G(t)$ with time for return flow: (a) at $\operatorname{Pr}=0.001, \mathrm{Bi}=1, k=4, \phi=90^{\circ}$ with various Reynolds numbers $R$; (b) at $\mathrm{Ma}=5, \mathrm{Pr}=0.001, \mathrm{Bi}=1, \phi=90^{\circ}$ with various wave numbers $k$; (c) at $\mathrm{Ma}=5, \mathrm{Pr}=0.001, \mathrm{Bi}=1, k=2$ with various propagation angles $\phi$; (d) at $R=3000, \mathrm{Bi}=10, k=3$, $\phi=90^{\circ}$ with various Prandtl numbers $\operatorname{Pr}$; (e) at $\mathrm{Ma}=3, \operatorname{Pr}=0.001, k=3, \phi=90^{\circ}$ with various Biot numbers Bi.

behaves like $O\left(R^{2}\right)$ [31]. However, in Figs. 2(a) and 3(a), we find that the relation of $G^{\text {max }}$ and $R$ is nearly linear. In Figs. 3(d) and 3(e), the variations of $G(t)$ with $\mathrm{Pr}$ and Bi are similar to those in linear flow in Figs. 2(d) and 3(e). 

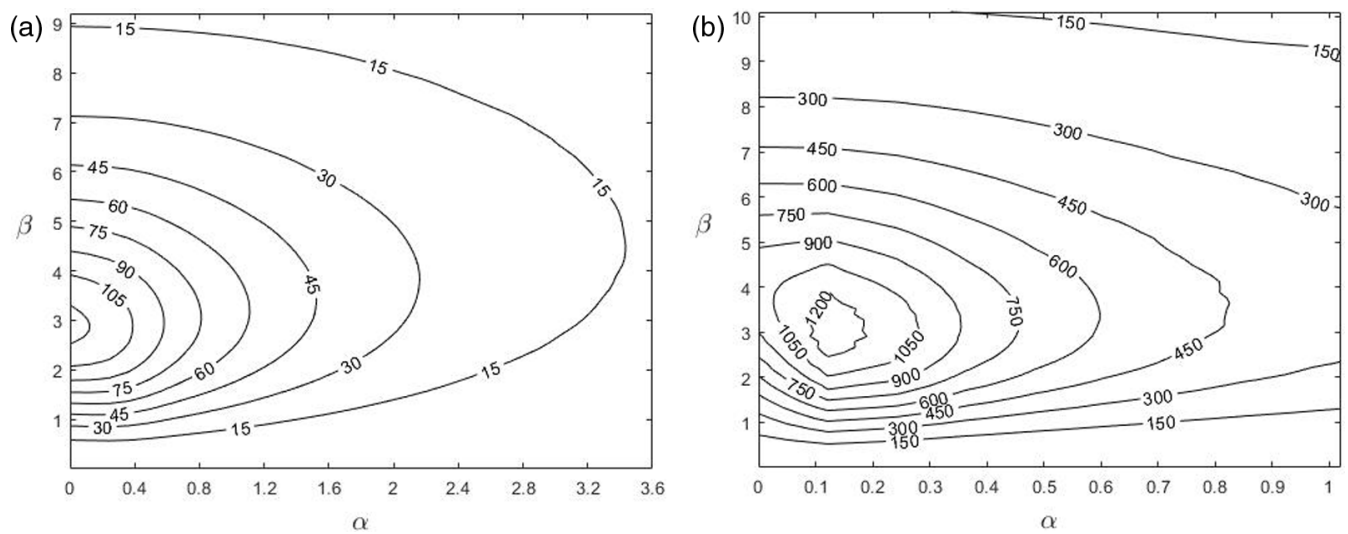

FIG. 4. Level lines of the maximum growth $G^{\max }$ in the $\alpha-\beta$ plane for linear flow at $\operatorname{Pr}=0.001$. (a) $\mathrm{Ma}=1, \mathrm{Bi}=0$, (b) $\mathrm{Ma}=4, \mathrm{Bi}=1$.

\section{B. Maximum growth}

The level lines of the maximum growth in the $\alpha-\beta$ plane for linear flow at $\operatorname{Pr}=0.001$ are displayed in Fig. 4. By modal analysis, we find that for $\mathrm{Bi}=0$, the critical Marangoni number at $\operatorname{Pr}=0.001$ is $\mathrm{Ma}_{\mathrm{c}} \approx 1.43$, while for $\mathrm{Bi}=1, \mathrm{Ma}_{\mathrm{c}} \approx 17$; these agree well with the results in Ref. [15]. Therefore the flows in Fig. 4 are subcritical. It can be seen that when $\mathrm{Ma}=1$, the optimal growth appears in the spanwise direction $(\alpha=0)$, which is similar to the case in plane Poiseuille flow [32]. However, for $\mathrm{Ma}=4$, the most amplified disturbance appears when $\phi$ is close to $90^{\circ}$, which is similar to the case in plane Couette flow [35]. The basic flow of linear flow is the same as that of plane Couette flow. However, the transient growth of the former is different from that of the latter. When $R=1000$, it can be seen in Fig. 4(a) that $G^{\text {opt }}=O(100)$; however, $G^{\text {opt }}=O(1000)$ for plane Couette flow [35]. In Fig. 4(b), the maximum growth is of the order of 1000 when $\mathrm{Ma}=4$ and $R=4000$. We can find that large transient growth $\left[G^{\max }=O(500)\right]$ still occurs even when $k=O(10)$.

The level lines of the maximum growth in the $\alpha-\beta$ plane for linear flow at $\operatorname{Pr}=0.01$ are displayed in Fig. 5. In modal analysis, $\mathrm{Ma}_{\mathrm{c}} \approx 4.43$ for $\mathrm{Bi}=0$, while $\mathrm{Ma}_{\mathrm{c}} \approx 14.5$ for $\mathrm{Bi}=1$. Therefore the flows in Fig. 5 are subcritical. It can be seen that the maximum growth in Fig. 5(a) is
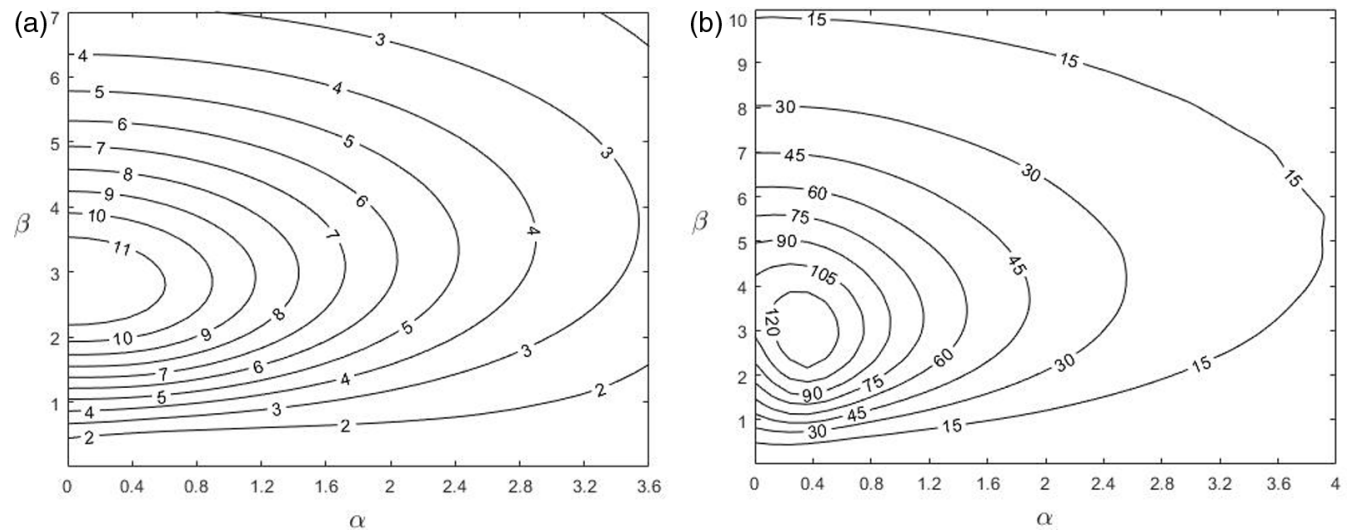

FIG. 5. Level lines of the maximum growth $G^{\max }$ in the $\alpha-\beta$ plane for linear flow at $\operatorname{Pr}=0.01$. (a) $\mathrm{Ma}=3$, $\mathrm{Bi}=0$, (b) $\mathrm{Ma}=12, \mathrm{Bi}=1$. 

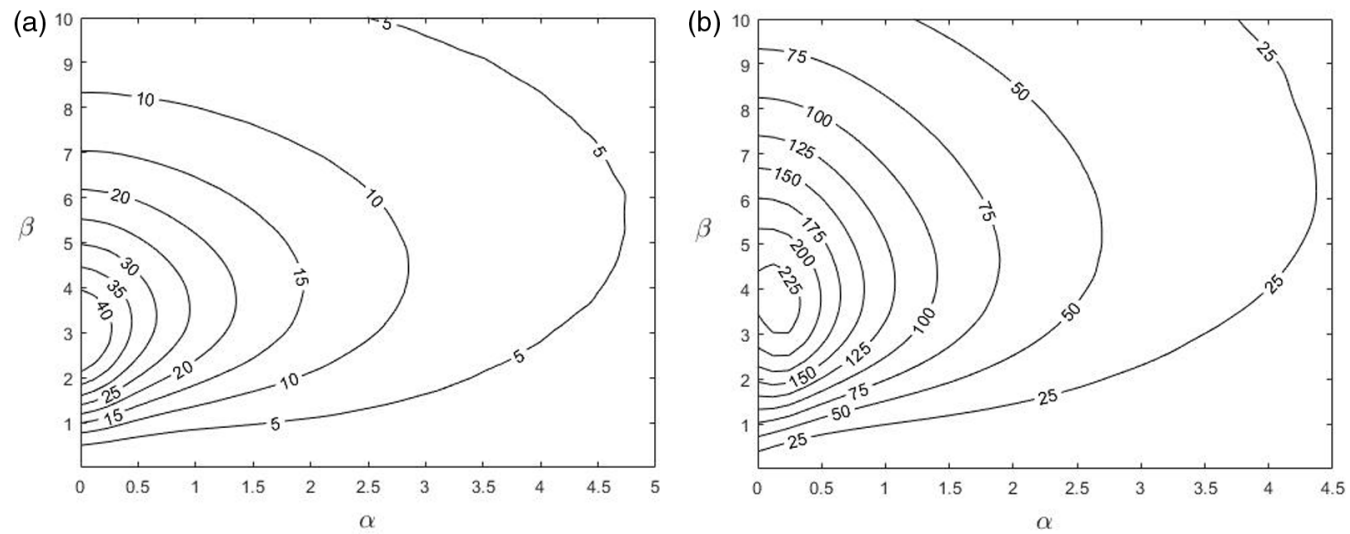

FIG. 6. Level lines of the maximum growth $G^{\max }$ in the $\alpha-\beta$ plane for return flow at $\operatorname{Pr}=0.001$. (a) $\mathrm{Ma}=1.5, \mathrm{Bi}=0$, (b) $\mathrm{Ma}=5, \mathrm{Bi}=1$.

far smaller than that in Fig. 4(a) even though Ma for the former is three times of that for the latter. In Fig. 5(b), $R=1200$, and the maximum growth has the same order as that in Fig. 4(a). This indicates that $G^{\max }$ is more sensitive to $R$. In Figs. 4 and 5, the optimal growth is reached when $\beta \approx 3$, which seems to be insensitive to $R$ and Pr.

The level lines of the maximum growth in the $\alpha-\beta$ plane for return flow at $\operatorname{Pr}=0.001$ and $\operatorname{Pr}=$ 0.01 are displayed in Figs. 6 and 7, respectively. In modal analysis, the critical values at $\operatorname{Pr}=0.001$ are $\mathrm{Ma}_{\mathrm{c}} \approx 1.97$ for $\mathrm{Bi}=0$, while $\mathrm{Ma}_{\mathrm{c}} \approx 7.2$ for $\mathrm{Bi}=1$. When $\mathrm{Pr}=0.01, \mathrm{Ma}_{\mathrm{c}} \approx 19.4$ for $\mathrm{Bi}=1$, while $\mathrm{Ma}_{\mathrm{c}} \approx 48$ for $\mathrm{Bi}=10$. Therefore the flows in Figs. 6 and 7 are subcritical. The optimal growth appears at $\alpha \approx 0, \beta \approx 3$ in Fig. 6(a), and $\alpha \approx 0.3, \beta \approx 4$ in Fig. 6(b). Comparing Fig. 6 with Fig. 7, we can find that the wave numbers in the $x$ and $y$ directions of the optimal growth change with Ma and Pr for return flow.

The level lines of the maximum time in the $\alpha-\beta$ plane corresponding to the cases in Figs. 4 and 6 at $\mathrm{Pr}=0.001, \mathrm{Bi}=1$ are displayed in Fig. 8. It can be seen that $t^{\text {max }}$ have the same order for a wide range of wave numbers. $t^{\mathrm{opt}}$ is not the maximum of $t^{\max }$ in the $\alpha-\beta$ plane. When $\alpha \geqslant 0.2, t^{\max }$ is not very sensitive to $\beta$. We find that the geometries of the contours of $G^{\max }$ and $t^{\max }$ at different $\operatorname{Pr}$ and Ma are qualitatively similar.
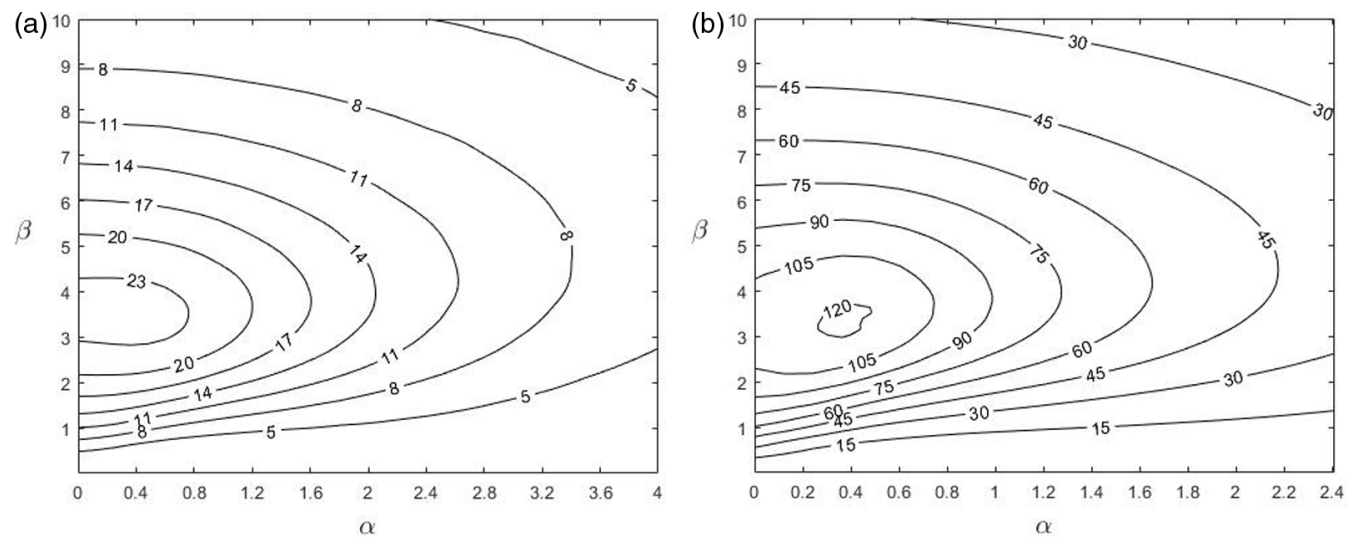

FIG. 7. Level lines of the maximum growth $G^{\max }$ in the $\alpha-\beta$ plane for return flow at $\operatorname{Pr}=0.01$. (a) $\mathrm{Ma}=$ $15, \mathrm{Bi}=1,(\mathrm{~b}) \mathrm{Ma}=35, \mathrm{Bi}=10$. 

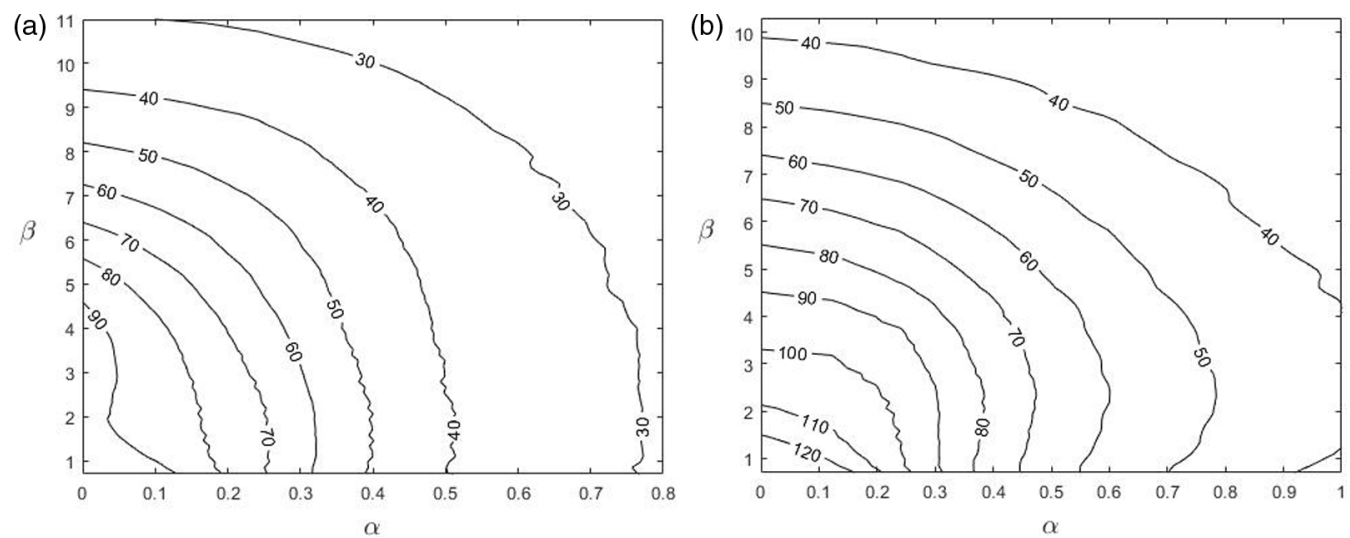

FIG. 8. Level lines of the maximum time $t^{\max }$ in the $\alpha-\beta$ plane at $\operatorname{Pr}=0.001, \mathrm{Bi}=1$ : (a) linear flow, $\mathrm{Ma}=4$; (b) return flow, $\mathrm{Ma}=5$.

\section{Perturbation flow field}

In this section, we pay attention to the flow field of the most amplified disturbance. The flow field is plotted in a new coordinate system $\left(x^{\prime}, y^{\prime}, z\right)$, where $x^{\prime}$ is the direction of the wave vector, $z$ is still the wall-normal direction, and $y^{\prime}$ is perpendicular to both of them. The velocities in the $x^{\prime}$ and $y^{\prime}$ directions are $u_{r}=u \cos \phi+v \sin \phi$ and $v_{r}=-u \sin \phi+v \cos \phi$, respectively. For the most amplified disturbance, $\phi \approx 90^{\circ}$. Thus, $u_{r} \approx v, v_{r} \approx-u$.

For plane shear flows, the streamwise streaks (narrow regions where the streamwise velocity is larger or smaller than the average) are observed in many experiments [40,41]. The nonmodal analysis of these flows [32,35] also suggested that there are streamwise streaks in the perturbation field which have the optimal growth. In the thermocapillary liquid layer, the case is similar to those in channel flows. The flow field of the most amplified disturbance for linear flow is displayed in Fig. 9. It can be seen that at the initial time and optimal time $t^{\text {max }}$, the flow fields are characterized by counter-rotating vortices and streaks. As $\alpha \ll \beta$, the vortices and streaks are nearly streamwise.

The amplitude of temperature perturbation at $t^{\max }$ is far larger than that at the initial time. The hot spots at $t^{\max }$ appear in the middle of the layer. The values of $\left|u_{r}\right|,\left|v_{r}\right|,|w|$, and $|T|$ show that (2.24) is satisfied, and the increase of the growth function is mainly caused by the increase of $\left|v_{r}\right|$. There is a little increase of $\left|u_{r}\right|$ at $t^{\max }$, while $|w|$ at $t^{\max }$ is smaller than the value at the initial time.

The flow field of the most amplified disturbance for return flow is displayed in Fig. 10. It can be seen that the hot spots are in the interior at $t^{\max }$. The maximum of $\left|v_{r}\right|$ is reached on the boundary of the counter-rotating vortices at $t^{\max }$, while the maximum of $\left|v_{r}\right|$ appears in the interior of the vortex at the initial time. The isolines of $\left|v_{r}\right|$ show the shapes of streaks. It is observed that the shapes of the streaks are tilted in Figs. 9 and 10, while those in plane Couette flow are upright [35]. The tilt direction at the initial time is opposite to that at $t^{\max }$.

\section{Energy analysis}

We study the energy mechanism in this section. The rate of change for perturbation kinetic energy can be written as follows [25,42],

$$
\begin{aligned}
\frac{\partial E_{\text {kin }}}{\partial t} & =-\frac{1}{2 R} \int(\boldsymbol{\tau}: \mathbf{S}) d^{3} r+\frac{1}{R} \int \mathbf{u} \cdot \boldsymbol{\tau} \cdot \mathbf{n} d^{2} r-\int \mathbf{u} \cdot\left[(\mathbf{u} \cdot \nabla) \mathbf{u}_{0}\right] d^{3} r \\
& =-N+M+I
\end{aligned}
$$

where $N$ is the work done by the perturbation stress, $M$ is the work done by Marangoni forces on the surface, and $I$ is the interaction between the perturbation flow and the basic flow. 

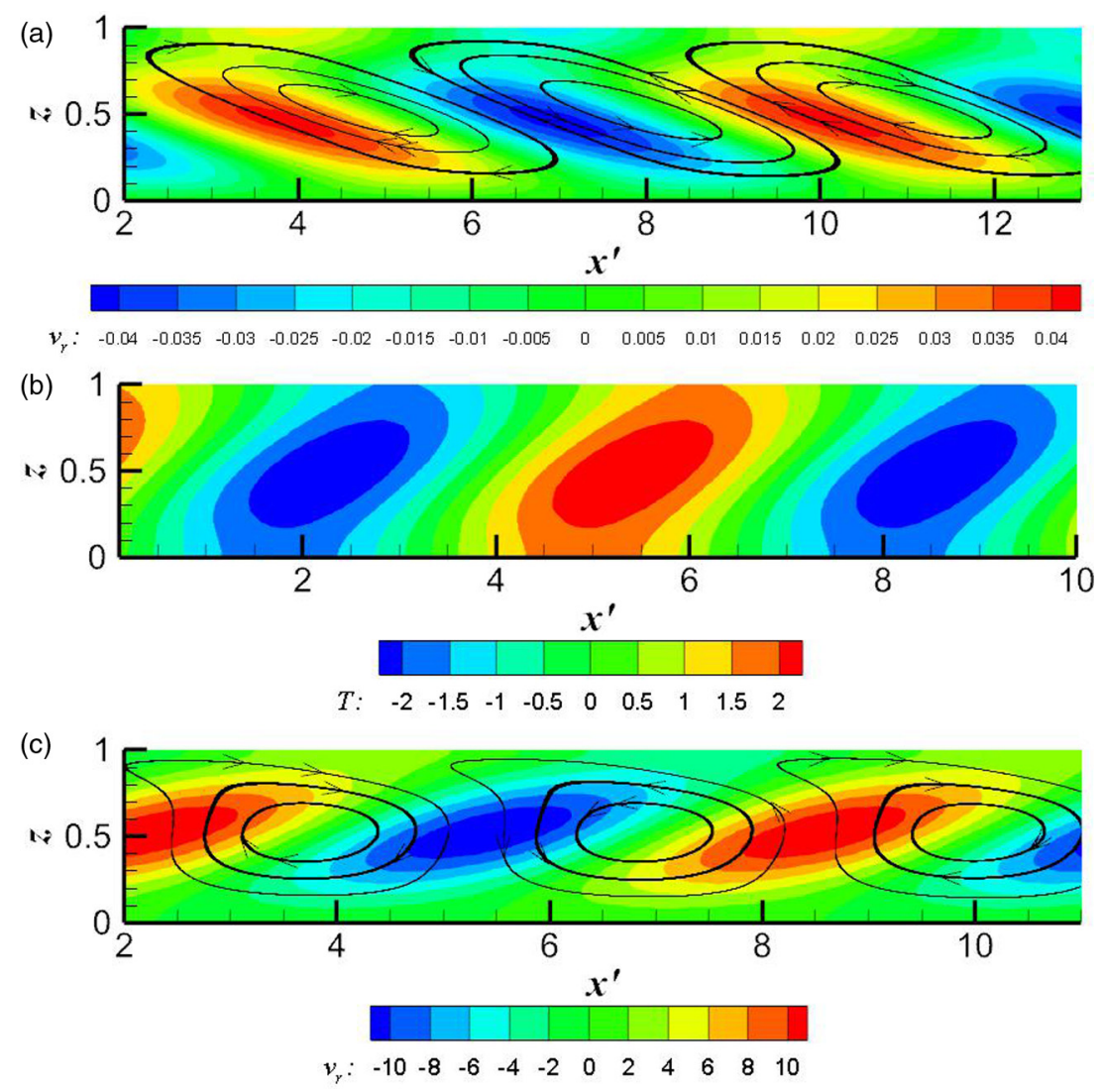

FIG. 9. The flow field of the most amplified disturbance for linear flow at $\operatorname{Pr}=0.01, \mathrm{Ma}=12, \mathrm{Bi}=1$, $\alpha=0.35, \beta=3$ : (a) the velocity field at the initial time; (b) the temperature field and (c) the velocity field at $t^{\max }=30.5$. In (a), (c), the streamlines of $\left(u_{r}, w\right)$ are plotted, while the color shows the value of $v_{r}$. The maximum growth is $G^{\max }=134$. At the initial time, the amplitudes of velocity and temperature are $\left|u_{r}\right|=$ $0.8139,\left|v_{r}\right|=0.0424,|w|=0.1642$, and $|T|=0.0147$, while at $t^{\max }=30.5,\left|u_{r}\right|=0.8633,\left|v_{r}\right|=11.8983$, $|w|=0.1373$, and $|T|=2.5135$.

For Newtonian fluid, the stress is proportional to the strain rate, so $N$ stands for the viscous dissipation $(N>0)$. The perturbation energy can only come from the last two terms in (3.3). It is known that $M$ is an important energy source for perturbation in modal stability analysis. For example, $M / I \geqslant 0.4$ at $\operatorname{Pr}=0.01$ [24]. In contrast, the energy of transient growth comes from the basic flow, which is similar to the mechanism of transient growth in channel flows.

In Tables II and III, the terms in (3.2) of the most amplified disturbance are listed, which are normalized by the kinetic energy,

$$
\int|\mathbf{u}|^{2} d^{3} r=1
$$

It can be found that $I$ decreases with $t$, while the viscous dissipation $N$ increases during the transient growth. The value of $M$ at $\operatorname{Pr}=0.01$ is larger than that at $\operatorname{Pr}=0.001$. However, $M$ is far smaller than the other two terms, so it is negligible for the energy mechanism. The transient growth only depends on the relative size of $I$ and $N . I>N$ when $t<t^{\max }$, so the perturbation kinetic energy increases with time. In contrast, $I<N$ when $t>t^{\max }$. This indicates that the growth function reaches its maximum at $t^{\max }$. 

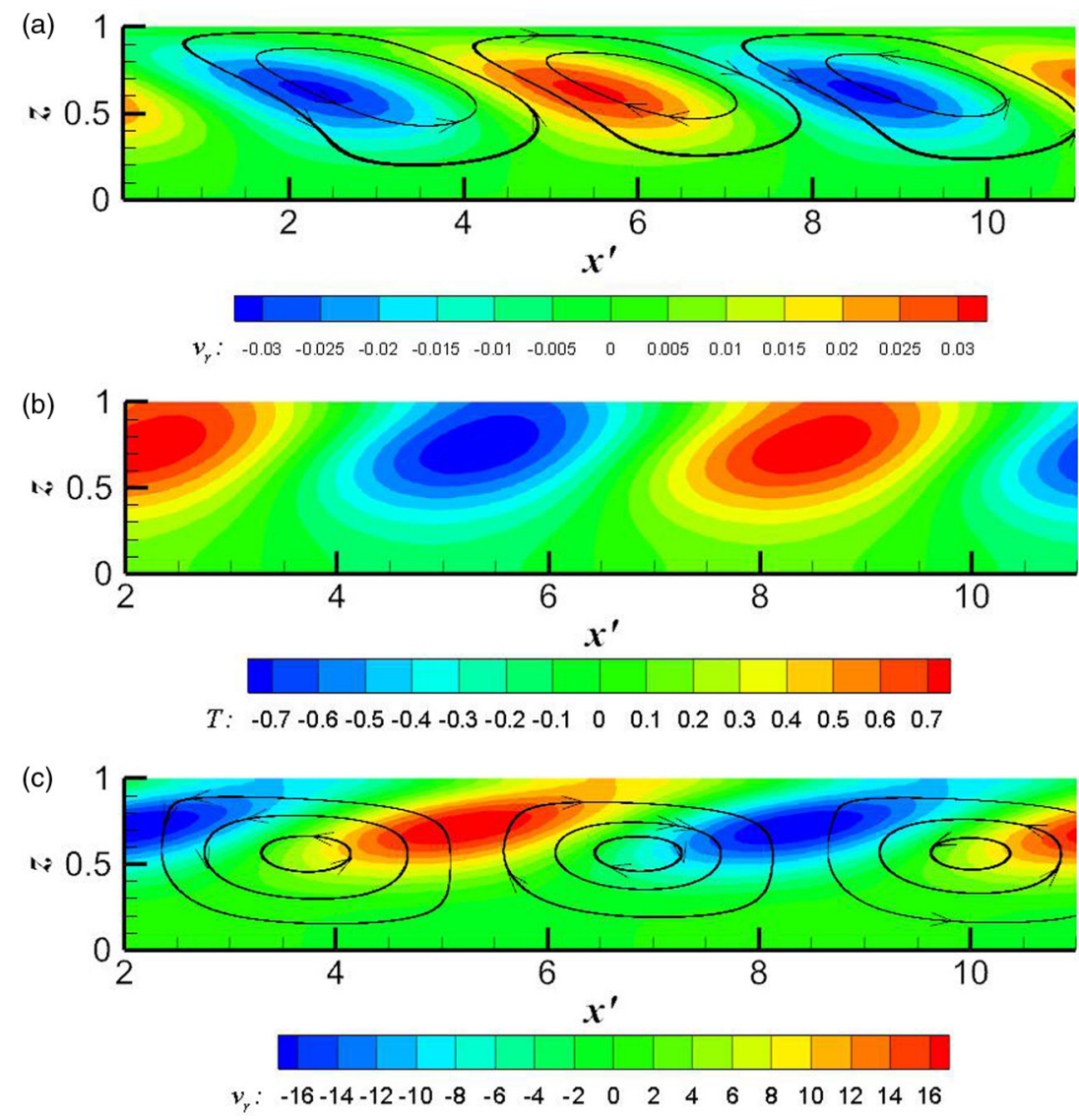

FIG. 10. The flow field of the most amplified disturbance for return flow at $\operatorname{Pr}=0.001, \mathrm{Ma}=5, \mathrm{Bi}=1$, $\alpha=0.2, \beta=4$ : (a) the velocity field at the initial time; (b) the temperature field and (c) the velocity field at $t^{\max }=91$. The maximum growth is $G_{\max }=235$. At the initial time, the amplitudes of velocity and temperature are $\left|u_{r}\right|=0.9489,\left|v_{r}\right|=0.0317,|w|=0.1737$, and $|T|=0.0019$, while at $t^{\max }=91,\left|u_{r}\right|=0.4322,\left|v_{r}\right|=$ $18.3395,|w|=0.1075$, and $|T|=0.8007$.

In Fig. 11, we plot the distribution of the energy from the basic low in the wall-normal direction. Here,

$$
\begin{aligned}
I & =-\int \mathbf{u} \cdot\left[(\mathbf{u} \cdot \nabla) \mathbf{u}_{0}\right] d^{3} r=\int P_{I} d z, \\
P_{I} & =-\int u\left(w D U_{0}\right) d^{2} r .
\end{aligned}
$$

TABLE II. The terms of perturbation kinetic energy growth in linear flow at $\operatorname{Pr}=0.01, \mathrm{Ma}=12, \mathrm{Bi}=1$, $\alpha=0.35, \beta=3, G^{\max } \approx 134, t^{\max } \approx 30.5$.

\begin{tabular}{lrrrrr}
\hline \hline$t$ & 15 & 25 & 30 & 35 & \multicolumn{1}{c}{45} \\
\hline$N$ & 0.014329 & 0.019808 & 0.024597 & 0.029659 & 0.038534 \\
$M$ & -0.000050 & 0.000104 & 0.000114 & 0.000089 & 0.000018 \\
$I$ & 0.074131 & 0.035298 & 0.026420 & 0.022000 & 0.019834 \\
$\frac{\partial E_{\text {Kin }}}{\partial t}$ & 0.059752 & 0.015594 & 0.001937 & -0.007570 & -0.018682 \\
\hline \hline
\end{tabular}


TABLE III. The terms of perturbation kinetic energy growth in return flow at $\mathrm{Pr}=0.001, \mathrm{Ma}=5, \mathrm{Bi}=1$, $\alpha=0.2, \beta=4, G^{\max } \approx 235.8, t^{\max } \approx 91$.

\begin{tabular}{lrcrrr}
\hline \hline$t$ & \multicolumn{1}{c}{40} & 70 & 90 & 120 & \multicolumn{1}{c}{160} \\
\hline$N$ & 0.005801 & 0.006685 & 0.007913 & 0.009983 & 0.011585 \\
$M$ & -0.000011 & 0.000003 & 0.000005 & 0.000004 & 0.000001 \\
$I$ & 0.024522 & 0.011695 & 0.008050 & 0.005958 & 0.006242 \\
$\frac{\partial E_{\text {kin }}}{\partial t}$ & 0.018710 & 0.005013 & 0.000142 & -0.004021 & -0.005342 \\
\hline \hline
\end{tabular}

It can be seen that the energy from the basic flow is related to $u$ and $w$, but is independent of $v$. In Fig. $11,\left|P_{I}\right| \approx 0$ near the boundaries, where the vertical velocity has $w=0 . P I>0$ in most regions. For return flow, the maximum of $P_{I}$ appears at $z \approx 0.75$, where $\left|D U_{0}\right|$ is relatively large. For linear flow, $D U_{0}=1$, and the maximum of $P_{I}$ appears in the middle of the layer. The distributions of $P_{I}$ at different times are similar.

\section{DISCUSSION}

We will discuss the mechanism of transient growth in thermocapillary liquid layers and make comparisons with those in channel flows and thermally driven flows in this section.

For channel flows, the transient growth is caused by the "lift-up" effect [31,43], where the streamwise streaks are amplified from streamwise vortices. The mechanism can be simply explained as follows. For large Reynolds numbers, the linearized momentum equation of streamwise perturbation velocity $u$ can be simplified as

$$
\frac{\partial u}{\partial t}+w D U_{0}=0
$$

During the transient growth, $w$ is nearly independent of time, so the streamwise velocity increases linearly with time.

A similar mechanism also exists in thermocapillary liquid layers. In Figs. 9(c) and 10(c), $u_{r} \approx$ $v, v_{r} \approx-u$, we can find that $\left|v_{r}\right|$ reaches its maximum on the boundary of vortices where $|w|$ is relatively large. For linear flow [see Fig. 9(c)], the maximum of $\left|v_{r}\right|$ appears in the middle of the layer, while for return flow, it appears at $z \approx 0.75$. This difference is due to $\left|D U_{0}\right|$, which has been discussed in Sec. III D.

(a)

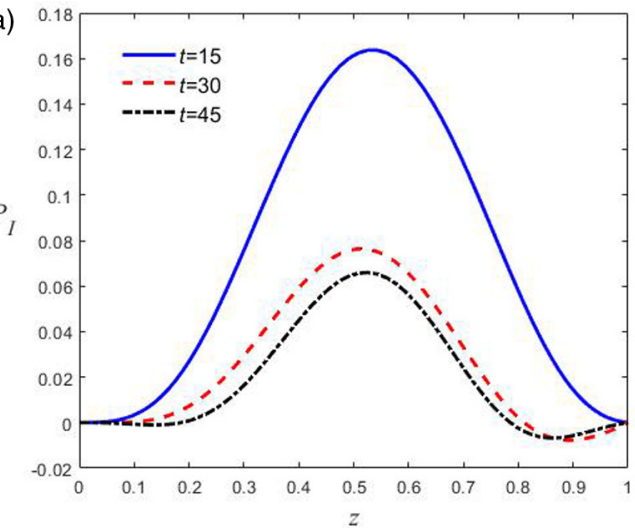

(b)

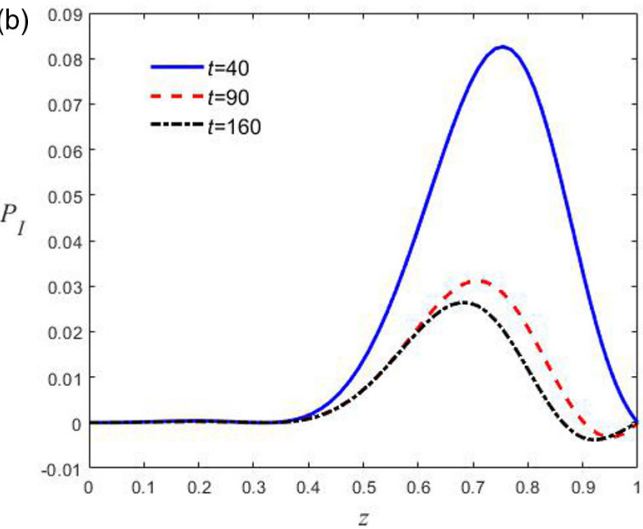

FIG. 11. The distribution of the energy from the basic flow in the wall-normal direction: (a) linear flow with the parameters in Table II; (b) return flow with the parameters in Table III. 
Besides the vortices and streaks, the temperature field also has a great effect on the transient growth in thermocapillary convections. For $R=1000, O\left(G^{\max }\right)=1000$ for plane Couette flow [35], while $O\left(G^{\max }\right)=100$ for linear flow, although the velocity profiles of them are the same. Furthermore, both $G^{\max }$ and $t^{\max }$ decrease with Pr [see Figs. 2(d) and Fig. 3(d)], but increase with Bi [see Figs. 2(e) and F3(e)]. The effect of temperature on the transient growth can be explained as follows.

Suppose the most amplified disturbance of plane Couette flow is added in the linear flow; then the vertical downward flow of vortices will increase the streamwise velocity. As the hot spot is mainly heated by the horizontal convection $\left(\widehat{u} \frac{\partial T_{0}}{\partial x}\right)$ for small Pr in thermocapillary liquid layers $[24,44]$, the increase of streamwise velocity can heat the flow region. Due to the heat conduction, the temperature of the surface above this region increases. However, the direction of Marangoni force induced by this temperature perturbation is opposite to the direction of velocity on the surface, so the kinetic energy will be decreased. Therefore the temperature field on the surface has a negative effect on the transient growth, and the maximum growth of linear flow is smaller than that of plane Couette flow. The heat flux to the gas is linearly related to $\mathrm{Bi}$, so the temperature perturbation on the surface becomes smaller when $\mathrm{Bi}$ increases. This is the reason why the maximum growth increases with $\mathrm{Bi}$ in Fig. 2(e).

There is rather large transient growth in subcritical flows at small Prandtl numbers. However, for moderate and high Prandtl numbers, the order of the critical Reynolds number is less than 1000 [15], and the energy mechanism shows that the energy of perturbation mainly comes from the work done by Marangoni forces on the surface, while the energy from the basic flow is very small [24]. Therefore there is no obvious transient growth at these Prandtl numbers.

We can find that $G^{\max }$ of return flow is obviously smaller than that of linear flow when they have the same Reynolds number (see Figs. 2 and 3). This may be due to the basic flow (see Fig. 1). The energy analysis in Sec. III D shows that the energy growth of perturbation mainly comes from the basic flow, and the energy from the basic flow is proportional to $D U_{0}$. For linear flow, $D U_{0}=1$, the energy mainly comes from the region $0.3 \leqslant z \leqslant 0.75$ [see Fig. 11(a)], while for return flow, $\left|D U_{0}\right|<1$ in most regions, the energy mainly comes from the region $0.6 \leqslant z \leqslant 0.85$ [see Fig. 11(b)]. Therefore there is less energy from the basic flow in return flow, and its transient growth is smaller.

To some extent, the thermocapillary liquid layer is similar to the thermally driven flows, such as Rayleigh-Bénard-Marangoni convection. In order to study the transient problem of the latter, some authors have used the frozen-time analysis $[45,46]$, where the unsteady basic state is supposed to evolve much more slowly than the perturbations [47]. On the contrary, the nonmodal analysis is widely used for instability problems of steady base flow [38], including the problem in this paper. Furthermore, the non-normal approach has been extended to transient cases by Doumenc et al. [47], which shows that the frozen-time assumption may fail in the transient problem while the non-normal approach is still valid. Therefore, the non-normal analysis may characterize the transition domain more properly.

\section{CONCLUSION}

We have performed the transient growth analysis for thermocapillary liquid layers by nonmodal stability theory. The maximum growth is determined for linear flow and return flow at different Prandtl numbers Pr. The results show that rather large transient growth occurs in subcritical flows at small Prandtl numbers, while the temperature field on the surface has a negative effect on the transient growth.

The transient growth function increases significantly with the Reynolds number $R$. Both the maximum growth $G^{\max }$ and time $t^{\max }$ decrease with Pr, but increase with the Biot number Bi. The optimal growth appears when the propagation angle is close to $90^{\circ}$ and the wave number is moderate $(k \approx 3-4)$. Comparing linear flow and return flow, we can find that the maximum growth of the former is obviously larger than that of the latter at the same Reynolds number. 
The flow field of the most amplified disturbance is characterized by counter-rotating vortices and streaks, which are both nearly streamwise. This is similar to the case in channel flows. However, the shapes of streaks are tilted in thermocapillary liquid layers, while those in plane Couette flow are upright. The increase of the transient growth function is mainly caused by the energy growth of streaks. For the temperature field, the amplitude of temperature perturbation at the maximum time $t^{\max }$ is far larger than that at the initial time, and the hot spots at $t^{\max }$ appear in the interior of the layer.

Energy analysis shows that the energy of transient growth at small Pr mainly comes from the basic flow, while the work done by Marangoni forces on the surface is negligible. For the most amplified disturbance, the energy from the basic flow $I$ decreases with time $t$, while the viscous dissipation $N$ increases during the transient growth. $I>N$ until $t$ reaches the maximum time $t^{\max }$. On the contrary, for moderate and high Prandtl numbers, the work done by Marangoni forces is dominant, while the energy from the basic flow is small. So the transient growth is not obvious at these Prandtl numbers.

\section{ACKNOWLEDGMENTS}

This work has been supported by the National Natural Science Foundation of China (Grants No.11872032, No. U1738119, and No. 11532015), and sponsored by K. C. Wong Magna Fund in Ningbo University.

[1] Crystal Growth Processes Based on Capillarity: Czochralski, Floating Zone, Shaping and Crucible Techniques, edited by T. Dufar (John Wiley \& Sons, New York, 2010).

[2] T. DebRoy and S. A. David, Physical processes in fusion welding, Rev. Mod. Phys. 67, 85 (1995).

[3] O. A. Basaran, H. Gao, and P. P. Bhat, Nonstandard inkjets, Annu. Rev. Fluid Mech. 45, 85 (2013).

[4] Q. Dai, M. M. Khonsari, C. Shen, W. Huang, and X. Wang, Thermocapillary migration of liquid droplets induced by a unidirectional thermal gradient, Langmuir 32, 7485 (2016).

[5] Y. Jiang and X. Zhou, Analysis of flow and heat transfer characteristics of nanofluids surface tension driven convection in a rectangular cavity, Int. J. Mech. Sci. 153, 154 (2019).

[6] S. H. Davis, Thermocapillary instabilities, Annu. Rev. Fluid Mech. 19, 403 (1987).

[7] M. F. Schatz and G. P. Neitzel, Experiments on thermocapillary instabilities, Annu. Rev. Fluid Mech. 33, 93 (2001).

[8] D. Kang, A. Nadim, and M. Chugunova, Marangoni effects on a thin liquid film coating a sphere with axial or radial thermal gradients, Phys. Fluids 29, 1 (2017).

[9] Z. Ding and T. N. Wong, Three-dimensional dynamics of thin liquid films on vertical cylinders with Marangoni effect, Phys. Fluids 29, 1 (2017).

[10] T. C. Kumawat and N. Tiwari, Hydrodynamic stability of thermoviscous liquid film inside a rotating horizontal cylinder: heating and cooling effects, Phys. Fluids 30, 1 (2018).

[11] D. Pettit, Saturday morning science videos, see https://spaceflight.nasa.gov/station/crew/exp6/ spacechronicles_videos.html (2003).

[12] B. Messmer, T. Lemee, K. Ikebukuro, I. Ueno, and R. Narayanan, Confined thermo-capillary flows in a double free-surface film with small Marangoni numbers, Int. J. Heat Mass Transfer 78, 1060 (2014).

[13] T. Yamamoto, Y. Takagi, Y. Okano, and S. Dost, Numerical investigation for the effect of the liquid film volume on thermocapillary flow direction in a thin circular liquid film, Phys. Fluids 25, 082108 (2013).

[14] T. Yamamoto, Y. Takagi, Y. Okano, and S. Dost, Numerical investigation of oscillatory thermocapillary flows under zero gravity in a circular liquid film with concave free surfaces, Phys. Fluids, 28, 032106 (2016).

[15] M. K. Smith and S. H. Davis, Instabilities of dynamic thermocapillary liquid layers. Part 1. Convective instabilities, J. Fluid Mech. 132, 119 (1983). 
[16] C. L. Chan and C. F. Chen, Effect of gravity on the stability of thermocapillary convection in a horizontal fluid layer, J. Fluid Mech. 647, 91 (2010).

[17] R. J. Riley and G. P. Neitzel, Instability of thermocapillary-buoyancy convection in shallow layers. Part 1. Characterization of steady and oscillatory instabilities, J. Fluid Mech. 359, 143 (1998).

[18] A. C. Or and R. E. Kelly, Thermocapillary and oscillatory-shear instabilities in a layer of liquid with a deformable surface. J. Fluid Mech. 360, 21 (1998).

[19] B. L. Smorodin, A. B. Mikishev, A. A. Nepomnyashchy, and B. I. Myznikova, Thermocapillary instability of a liquid layer under heat flux modulation, Phys. Fluids 21, 062102 (2009).

[20] S. Yang, R. Liang, and J. He, Oscillating characteristic of free surface from stability to instability of thermocapillary convection for high Prandtl number fluids, Int. J. Heat Fluid Flow 61, 298 (2016).

[21] L. Zhang, Y. R. Li, C. M. Wu, and L. Zhang, Effect of surface heat dissipation on thermocapillary convection of moderate Prandtl number fluid in a shallow annular pool, J. Cryst. Growth 514, 21 (2019).

[22] L. A. Davalos-Orozco, Sideband thermocapillary instability of a thin film flowing down the outside of a thick walled cylinder with finite thermal conductivity, Int. J. Non-Linear Mech. 109, 15 (2019).

[23] Q. Kang, J. Wang, L. Duan, Y. Su, J. He, D. Wu, and W. Hu, The volume ratio effect on flow patterns and transition processes of thermocapillary convection, J. Fluid Mech. 868, 560 (2019).

[24] C. Y. Yan, K. X. Hu, and Q. S. Chen, Thermocapillary instabilities of liquid layers on an inclined plane, Phys. Fluids 30, 082101 (2018).

[25] K. X. Hu, M. He, and Q. S. Chen, Instability of thermocapillary liquid layers for Oldroyd-B fluid, Phys. Fluids 28, 033105 (2016).

[26] K. Hu, M. He, Q. Chen, and R. Liu, Effect of gravity on the stability of viscoelastic thermocapillary liquid layers, Int. J. Heat Mass Transfer 123, 776 (2018).

[27] K. Hu, M. He, Q. Chen, and R. Liu, Linear stability of thermocapillary liquid layers of a shear-thinning fluid, Phys. Fluids 29, 073101 (2017).

[28] K. Hu, M. He, Q. Chen, and R. Liu, On the stability of thermocapillary convection of a Bingham fluid in an infinite liquid layer, Int. J. Heat Mass Transfer 112, 993 (2018).

[29] P. J. Schmid, Nonmodal stability theory, Annu. Rev. Fluid Mech. 39, 129, (2007).

[30] R. R. Kerswell, Nonlinear nonmodal stability theory, Annu. Rev. Fluid Mech. 50, 319,(2017).

[31] L. N. Trefethen, A. E. Trefethen, S. C. Reddy, and T. A. Driscoll, Hydrodynamic stability without eigenvalues, Science 261, 578 (1993).

[32] S. C. Reddy and D. S. Henningson, Energy growth in viscous channel flows, J. Fluid Mech. 252, 209 (1993).

[33] P. J. Schmid and D. S. Henningson, Optimal energy density growth in Hagen-Poiseuille flow, J. Fluid Mech. 277, 197 (1994).

[34] C. Nouar, N. Kabouya, J. Dusek, and M. Mamou, Modal and non-modal linear stability of the plane Bingham-Poiseuille flow, J. Fluid Mech. 577, 211 (2007).

[35] R. Liu and Q. S. Liu, Non-modal instability in plane Couette flow of a power-law fluid, J. Fluid Mech. 676, 145 (2011).

[36] G. Balestra, P. T. Brun, and F. Gallaire, Rayleigh-Taylor instability under curved substrates: An optimal transient growth analysis, Phys. Rev. Fluids 1, 083902 (2016).

[37] Y. R. Li, N. Imaishi, T. Azami, and T. Hibiya, Three-dimensional oscillatory flow in a thin annular pool of silicon melt, J. Cryst. Growth 260, 28 (2004).

[38] P. J. Schmid and D. S. Henningson, Stability and Transition in Shear Flows (Springer, Berlin, 2001).

[39] A. Sameen and R. Govindarajan, The effect of wall heating on instability of channel flow, J. Fluid Mech 577, 417 (2007).

[40] S. J. Kline, W. C. Reynolds, F. A. Schraub, and P. W. Runstadler, The structure of turbulent boundary layers, J. Fluid Mech. 30, 741 (1967).

[41] O. Kiton, K. Nakabyashi, and F. Nishimura, Experimental study on mean velocity and turbulence characteristics of plane Couette flow: Low-Reynolds-number effects and large longitudinal vortical structure, J. Fluid Mech. 539, 199 (2005).

[42] K. X. Hu, J. Peng, and K. Q. Zhu, The linear stability of plane Poiseuille flow of Burgers fluid at very low Reynolds numbers, J. Non-Newtonian Fluid Mech. 167-168, 87 (2012). 
[43] M. T. Landahl, A note on an algebraic instability of inviscid parallel shear flows, J. Fluid Mech. 98, 243 (1980).

[44] M. K. Smith, Instability mechanisms in dynamic thermocapillary liquid layers, Phys. Fluids 29, 3182 (1986).

[45] P. M. Gresho and R. L. Sani, The stability of a fluid layer subjected to a step change in temperature: Transient vs. frozen time analyses, Int. J. Heat Mass Transfer 14, 207 (1971).

[46] K. H. Kang and C. K. Choi, A theoretical analysis of the onset of surface-tension-driven convection in a horizontal liquid layer cooled suddenly from above, Phys. Fluids 9, 7 (1997).

[47] F. Doumenc, T. Boeck, B. Guerrier, and M. Rossi, Transient Rayleigh-Bénard-Marangoni convection due to evaporation: A linear non-normal stability analysis, J. Fluid Mech. 648, 521 (2010). 